\title{
BRÉZIS-GALLOUËT-WAINGER INEQUALITY WITH A DOUBLE LOGARITHMIC TERM ON A BOUNDED DOMAIN AND ITS SHARP CONSTANTS
}

\author{
Kei Morit, Tokushi Sato And Hidemitsu Wadade
}

Abstract. The Brézis-Gallouët-Wainger inequality gives an estimate of the $L^{\infty}$-norm by the critical Sobolev norm with the aid of the logarithmic dependency of a higher order Sobolev norm. We investigate the Brézis-Gallouët-Wainger inequality on a bounded domain with the first order critical Sobolev space, and give the best constant in the inequality in some special cases. Furthermore, since the inequality does not hold with the sharp constant, we add a double logarithmic term and give the sharp constant for its coefficient. A part of our results is mainly based on an investigation of the inequality with the higher-order Sobolev norm replaced by the Hölder seminorm.

Mathematics subject classification (2010): 46E35.

Keywords and phrases: Sobolev embedding theorem, Brézis-Gallouët-Wainger inequality, sharp constants, double logarithmic term.

\section{REFERENCES}

[1] R. A. Adams and J. J. F. Fournier, Sobolev spaces, Second edition, Pure and Applied Mathematics, Vol. 140, Academic Press, New York-London, 2003.

[2] H. BrÉzis And T. Gallouët, Nonlinear Schrödinger evolution equations, Nonlinear Anal., 4, 4 (1980), 677-681.

[3] H. BRÉZIS AND S. WAINGER, A note on limiting cases of Sobolev embeddings and convolution inequalities, Comm. Partial Differential Equations, 5, 7 (1980), 773-779.

[4] S. Ibrahim, M. Majdoub And N. MASMOUdi, Double logarithmic inequality with a sharp constant, Proc. Amer. Math. Soc., 135, 1 (2007), 87-97.

[5] K. MORII, T. SATO AND H. WADADE, Brézis-Gallouët-Wainger type inequality with a double logarithmic term in the Hölder space: its sharp constants and extremal functions, to appear in Nonlinear Anal. 